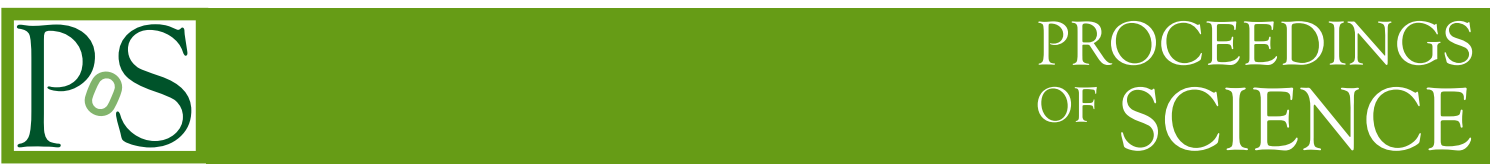

\title{
Properties of non-local wave function equivalent potential with generalized derivative expansion
}

\author{
Takuya Sugiura ${ }^{* a}$, Keiko Murano $^{a}$, Noriyoshi Ishii $^{a}$, and Makoto Oka ${ }^{b c}$ \\ ${ }^{a}$ Research Center for Nuclear Physics(RCNP), Osaka University, Osaka 567-0047, Japan \\ ${ }^{b}$ Department of Physics, Tokyo Institute of Technology, Tokyo 152-8551, Japan \\ ${ }^{c}$ Advanced Science Research Center, Japan Atomic Energy Agency, Tokai, Ibaraki, 319-1195, \\ Japan \\ E-mail: sugiura@rcnp.osaka-u.ac.jp
}

We examine the properties of the wave-function-equivalent potentials which HAL QCD collaboration has introduced. We generalize the derivative expansion, and then apply it to energyindependent and non-local potentials in a coupled-channel model. We observe that the expansion converges by comparing the scattering phase shifts computed from these potentials with the exact one. We have also found that the convergence can be improved by either varying the choice of interpolating fields or tuning the expansion scale in the generalized derivative expansion. The results will be utilized in future lattice QCD calculations, allowing for further application of the HAL QCD method.

34th annual International Symposium on Lattice Field Theory

24-30 July 2016

University of Southampton, UK

* Speaker. 


\section{Introduction}

Deriving the nuclear force from QCD is one of the most important subjects in nuclear physics, while it has been a challenge because of the non-perturbative nature of low-energy QCD. Recently, a method to determine the nuclear force through lattice QCD calculations has been introduced and vigorously developed by HAL QCD collaboration $[1,2]$. The method is based on the use of the Nambu-Bethe-Salpeter (NBS) wave functions as inputs to the Schrödinger equation, so that the nuclear force is determined as a non-local ${ }^{1}$ and energy-independent potential which reproduces all the NBS wave functions simultaneously. The non-locality of the HAL QCD potential is taken into account by the derivative expansion, so that the potential is expressed as a power series of spatial derivatives whose coefficients are determined from the NBS wave functions for different energies. In a previous study [3], the authors studied the validity of the derivative expansion in an indirect method: they compared two local lowest-order HAL QCD potentials computed from the NBS wave functions for c.m. energies $E \sim 0 \mathrm{MeV}$ and $E \sim 45 \mathrm{MeV}$. The two states appeared as the ground states with the periodic and the anti-periodic spatial boundary conditions. They found that the potentials agreed within statistical errors. However, it is desirable to evaluate the derivative expansion more explicitly, since the higher-order terms might not be negligible for higher-energy nucleon-nucleon scattering.

We perform a model calculation to examine the properties of the HAL QCD potentials when the higher-order terms of the derivative expansion are explicitly taken into account. We also study the possibility of improving the convergence by either varying the choice of interpolating fields or introducing a non-locality scale parameter to the expansion.

\section{Model}

We employ a 1+1 dimensional coupled-channel model originally proposed by M. Birse [4]. We first construct a second-quantized non-relativistic Hamiltonian $\hat{H}$, involving three different scalar fields, $\hat{p}(x), \hat{n}(x)$, and $\hat{n}^{\prime}(x)$, which are analogous to proton, neutron, and the first excited state of neutron with excitation energy $\Delta$, respectively. We consider an attractive square-well interaction

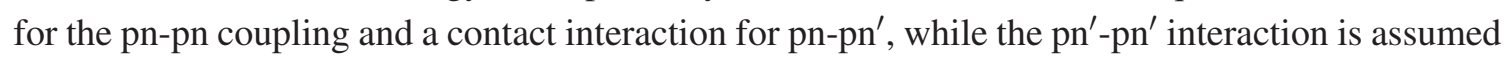
to be absent for simplicity. To discuss the two-body scattering problem in this system, we consider the two-particle states of $\mathrm{pn}$ and $\mathrm{pn}^{\prime}$. We define the wave functions for these channels as

$$
\psi_{0}(x) \equiv\langle 0|\hat{p}(x+y) \hat{n}(y)| \Psi(E)\rangle, \quad \psi_{1}(x) \equiv\left\langle 0\left|\hat{p}(x+y) \hat{n}^{\prime}(y)\right| \Psi(E)\right\rangle,
$$

where $|\Psi(E)\rangle$ denotes the energy eigenstate in the center of mass frame satisfying $\hat{H}|\Psi(E)\rangle=$ $E|\Psi(E)\rangle$. We then find the wave functions satisfy the following coupled-channel equations:

$$
\begin{gathered}
{\left[-\frac{1}{M} \frac{d^{2}}{d x^{2}}+V_{0}(x)-E\right] \psi_{0}(x)+2 g \delta(x) \psi_{1}(x)=0,} \\
{\left[-\frac{1}{M} \frac{d^{2}}{d x^{2}}+\Delta-E\right] \psi_{1}(x)+2 g \delta(x) \psi_{0}(x)=0,}
\end{gathered}
$$

\footnotetext{
${ }^{1}$ In this paper, we use the term non-local for the potentials that cannot be parameterized as $\left\langle x|\hat{V}| x^{\prime}\right\rangle=V(x) \delta\left(x-x^{\prime}\right)$ in the coordinate space.
} 
with

$$
V_{0}(x)=\left\{\begin{array}{lll}
-V_{0} & \text { for } & |x|<R \\
0 & \text { for } & |x|>R .
\end{array}\right.
$$

The even-parity solution of Eqs. (2.2) is obtained analytically, which is conveniently utilized to implement the derivative expansion with precision. We exclusively focus on the energy region $E<\Delta$ so that $\psi_{1}(x)$ vanishes at large distance.

In this model, a general interpolating field for "neutron" is achieved as a linear combination of the fields $n(x)$ and $n^{\prime}(x)$. We define such a field according to

$$
\hat{\phi}_{q}(x) \equiv \hat{n}(x)+q \hat{n}^{\prime}(x)
$$

where a real parameter $q$ is introduced to arrange the mixing of the states. We refer to $q$ as the field admixture parameter in this paper. The NBS wave function with the fields $\hat{p}(x)$ and $\hat{\phi}_{q}(x)$ is expressed as a linear combination of $\psi_{0}(x)$ and $\psi_{1}(x)$ :

$$
\Psi_{q}(x) \equiv\left\langle 0\left|\hat{p}(x+y) \hat{\phi}_{q}(y)\right| \Psi(E)\right\rangle=\psi_{0}(x)+q \psi_{1}(x) .
$$

We utilize $\Psi_{q}(x)$ to construct HAL QCD potentials, and the dependence on the choice of interpolating fields is studied by varying the field admixture parameter $q$.

The contribution of $\psi_{1}(x)$ is suppressed at large distance, so that the long-distance asymptotic behavior of our NBS wave function reads

$$
\Psi_{q}(x) \rightarrow \psi_{0}(x) \simeq A \cos (k|x|+\delta(k)),
$$

where $\delta(k)$ denotes the scattering phase shift and $k \equiv \sqrt{M E}$. Similar relation for the NBS wave function on the lattice has been derived [2] through the LSZ reduction formula. This relation ensures that the HAL QCD potentials are faithful to the scattering phase shift by construction.

\section{Non-Local Potential and Derivative Expansion}

Now we discuss how to construct a potential by using the NBS wave functions (2.5) for various different energies. We start from the following Schrödinger equation in a finite box:

$$
\left(-H_{0}+E_{m}\right) \Psi\left(x ; E_{m}\right)=\int d x^{\prime} V\left(x, x^{\prime}\right) \Psi\left(x^{\prime} ; E_{m}\right),
$$

where $H_{0} \equiv-\frac{1}{M} \frac{d^{2}}{d x^{2}}$ is the free Hamiltonian and $E_{m}$ are the discrete energy eigenvalues. In general, the potential has to be non-local to reproduce a given set of NBS wave functions $\Psi\left(x ; E_{m}\right)$ for different energies. The non-locality can be taken into account by the (naïve) derivative expansion:

$$
V\left(x, x^{\prime}\right)=\sum_{n=0}^{\infty} u_{n}(x)\left(\frac{\partial}{\partial x}\right)^{n} \delta\left(x-x^{\prime}\right),
$$

where the derivatives in the higher order terms yield the non-locality.

The naïve derivative expansion (3.2) does not work well in the Birse model. With the naïve expansion, Eq. (3.1) is rewritten as

$$
\left(-H_{0}+E_{m}\right) \Psi\left(x ; E_{m}\right)=\sum_{n=0}^{\infty} u_{n}(x) \frac{d^{n} \Psi}{d x^{n}}\left(x ; E_{m}\right) .
$$


We find that $\frac{d^{n} \Psi}{d x^{n}}$ on the right-hand side are singular at $x=0$ and $x= \pm R$, because of the $\delta(x)$ coupling and the square-well edges in Eqs. (2.2), respectively. To avoid the singularity, we introduce a generalized derivative expansion as follows:

$$
V\left(x, x^{\prime}\right)=\sum_{n=0}^{\infty} v_{n}(x)\left(\frac{\partial}{\partial x}\right)^{n} \delta_{\rho}\left(x-x^{\prime}\right), \quad \delta_{\rho}\left(x-x^{\prime}\right) \equiv \frac{\exp \left\{-\left(x-x^{\prime}\right)^{2} / \rho^{2}\right\}}{\sqrt{\pi} \rho}
$$

where $\rho$ is an arbitrary real parameter. In the following, we refer to $\rho$ as the Gaussian expansion scale. In the case of the generalized expansion, Eq. (3.1) is rewritten as

$$
\left(-H_{0}+E_{m}\right) \Psi\left(x ; E_{m}\right)=\sum_{n=0}^{\infty} v_{n}(x) \frac{d^{n} \Phi_{\rho}}{d x^{n}}\left(x ; E_{m}\right),
$$

where the smeared wave function $\Phi_{\rho}\left(x ; E_{m}\right) \equiv \int d x^{\prime} \delta_{\rho}\left(x-x^{\prime}\right) \Psi\left(x^{\prime} ; E_{m}\right)$ appearing on the righthand side is smooth everywhere so that it causes no singularity. In Ref. [5], we give a proof that the generalized derivative expansion is exact. It is a natural generalization of the naïve derivative expansion, since $\delta_{\rho}\left(x-x^{\prime}\right)$ is reduced to $\delta\left(x-x^{\prime}\right)$ in the $\rho \rightarrow 0$ limit. Moreover, we expect that the convergence of the generalized expansion can be improved by tuning the expansion scale $\rho$. We have made a few non-essential and technical modifications to Eq. (3.4) in presenting the results below. The details are found in Ref. [5].

In order to examine the convergence, we first truncate the summation over $n$ in Eq. (3.4) at a finite order, $N$. The coefficient functions $v_{n}(x)(n=0, \cdots, N)$ are then determined from the NBS wave functions (2.5) for the $(N+1)$ lowest-lying energy levels in a finite box. Because of the $\delta(x)$ coupling terms in Eq. (2.2), each coefficient $v_{n}(x)$ shall be decomposed into a regular part $\tilde{v}_{n}(x)$ and the $\delta$ functional singular part as $v_{n}(x)=\tilde{v}_{n}(x)+g_{n} \delta(x)$. The singular part is treated separately to determine the strength constants $g_{n}$ from the same NBS wave functions. We then solve the Lippmann-Schwinger equation with these HAL QCD potentials to calculate the scattering phase shift defined as Eq. (2.6). The result is compared to the analytic solution of the model to discuss the convergence of the expansion.

\section{Results}

We employ the parameter set given in Ref. [4], i.e. $M V_{0}=1 / R^{2}, M \Delta=6 / R^{2}, M g=6 / R$, and we take $R=1$ and $M=1$. The solution of coupled-channel Eqs. (2.2) is analytically obtained under the twisted boundary condition (TBC)

$$
\begin{aligned}
\psi_{0,1}(x+2 L) & =e^{i \theta} \psi_{0,1}(x), \\
\psi_{0,1}^{*}(x) & =\psi_{0,1}(-x),
\end{aligned}
$$

with $L=10$ and $\theta=\pi / 2$. The second condition implies that the real part of the solution is parityeven and the imaginary part is parity-odd. Thus we only use the real part to consider the parityeven sector. The application of the commonly used (anti-)periodic BC leads to a technical problem around the boundary, since the even-order (odd-order) derivatives of the NBS wave functions would be zero for all energies. With this parameter set and the TBC, we find a bound state at $E=E_{0} \simeq$ -33.7 and 15 excited states, $E=E_{1}, \cdots, E_{15}$ below the threshold $E=\Delta$. A HAL QCD potential with truncation order $N$ is determined through the Schrödinger equations for $E=E_{0}, \cdots, E_{N}$. 
In Fig. 1, we show the regular parts of the HAL QCD potentials with truncation orders $N=$ $1,2,3,4,5$ and fixed $q=+0.2$ and $\rho=0.5$. We see that the structure of the potentials varies depending on the truncation order, as the magnitude tends to become larger for larger $N$. All the potentials are finite-ranged, and we find $V\left(x, x^{\prime}\right) \simeq 0$ for $|x|>3$; therefore, we safely regard $V\left(x, x^{\prime}\right)=0$ for $|x|>4$ in calculating the phase shift.

(a) : $\mathrm{N}=1$

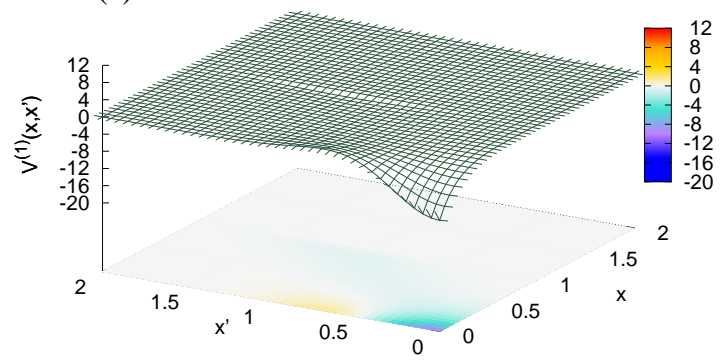

(c) : $\mathrm{N}=3$

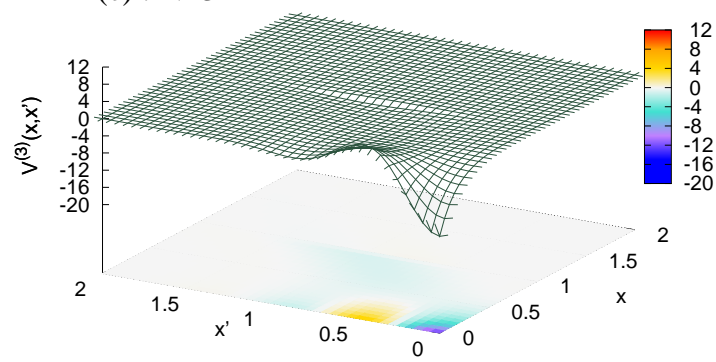

(e) : N=5

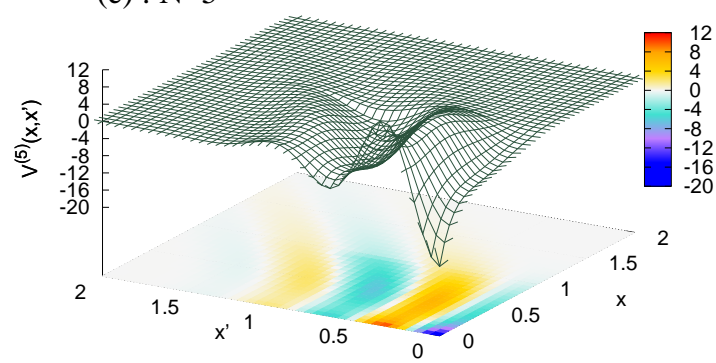

(b) : N=2

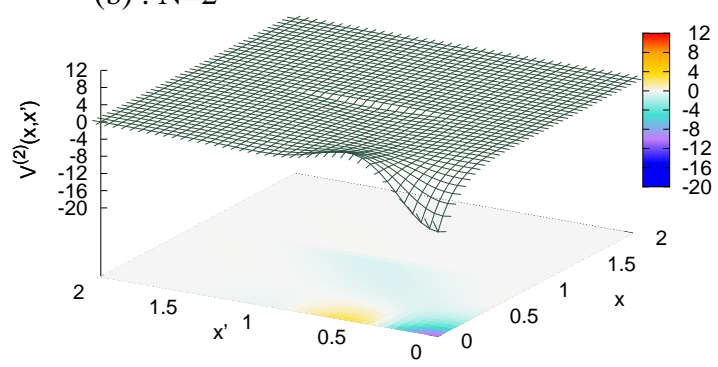

(d) : $\mathrm{N}=4$

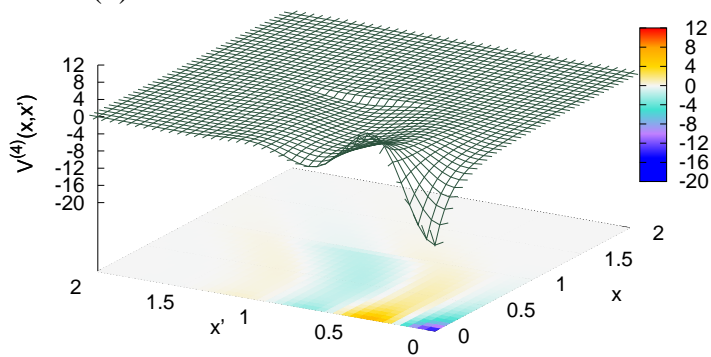

Figure 1: Non-local potentials $V\left(x, x^{\prime}\right)$ with truncation orders of (a) $N=1$, (b) $N=2$, (c) $N=3$, (d) $N=4$, and (e) $N=5$. The field adxmixture parameter and the Gaussian expansion scale are fixed to $q=+0.2$ and $\rho=0.5$, respectively.

Figure 2 shows the scattering phase shifts $\delta_{N}(E)$ computed from the HAL QCD potentials in Fig. 1, i.e., the ones with fixed $q=+0.2$ and $\rho=0.5$ and varying truncation orders $N=1, \cdots, 5$. The exact phase shift $\delta_{\text {exact }}(E)$ extracted from the analytic solution is also plotted for comparison. We see that each of $\delta_{N}$ reproduces $\delta_{\text {exact }}$ in a small energy region, but deviates away for the higher energies. However, the deviation becomes smaller as $N$ increases and more terms are included. It indicates that the generalized derivative expansion actually converges to give the correct phase shift. 
The agreement between $\delta_{N}$ and $\delta_{\text {exact }}$ for $E \leq E_{N}$ is ensured by construction, since the NBS wave functions for these energies are used as inputs to the Schrödinger equation. The result also shows that the agreement extends beyond these energies, indicating that extrapolation to higher energies is possible. In the following, we refer to such a region with nontrivial agreement as the extrapolation region, and use it to discuss the convergence.

Note that our result in the $1+1$ dimensional space-time shows the relation $\delta(E=0)=\pi / 2$ with the existence of a single bound state, which is contrasted to $\delta_{l}(E=0)-\delta_{l}(E=\infty)=n_{l} \pi$ in $1+3$ dimension with $n_{l}$ bound states of angular momentum $l$.

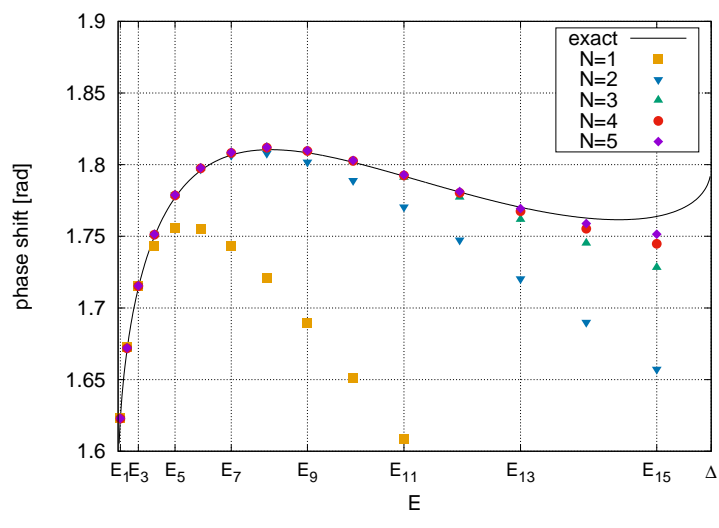

Figure 2: Scattering phase shifts $\delta_{N}(E)$ at truncations $N=1,2,3,4,5$, compared to the exact one $\delta_{\text {exact }}(E)$ extracted from the analytic solution of the model. Here $q=+0.2$ and $\rho=0.5$ are fixed.

To study the dependence on the choice of interpolating fields, we vary the field admixture parameter $q$. In Fig. 3, we show the phase shifts with $q=-1.0,+0.2$ and fixed $\rho=0.5$ at truncation orders $N=2,3$. We see that the derivative expansion is also convergent with $q=-1.0$, since the result with $(q, N)=(-1.0,3)$ shows better agreement with the exact one than that of $(q, N)=$ $(-1.0,2)$. Moreover, we see that the upper limit of the extrapolation region is comparable between the $(q, N)=(-1.0,3)$ and $(q, N)=(+0.2,2)$ cases, despite the different truncation orders. It implies that the convergence can be improved by tuning the choice of interpolating fields.

We expect the Gaussian expansion scale $\rho$ can also be used to improve the convergence of the generalized derivative expansion. In Fig. 4, we show the scattering phase shifts with $\rho=$ $0.3,0.5$ and $N=2,3$, while the field admixture parameter is fixed to $q=+0.2$. The convergence of the generalized expansion is confirmed once again for each choice of $\rho$. The upper limit of the extrapolation region in the case of $(\rho, N)=(0.3,2)$ is shown to be similar to that of $(\rho, N)=$ $(0.7,3)$, although the truncation order is smaller and thus fewer number of NBS wave functions are used as inputs. We therefore observe that we can improve the convergence by properly choosing the expansion scale in the generalized derivative expansion, as well as tuning interpolating fields. In practice, this property will be of great use, since the generalized derivative expansion is also applicable to lattice QCD calculations, and varying the $\rho$ value does not require any additional computational cost. 


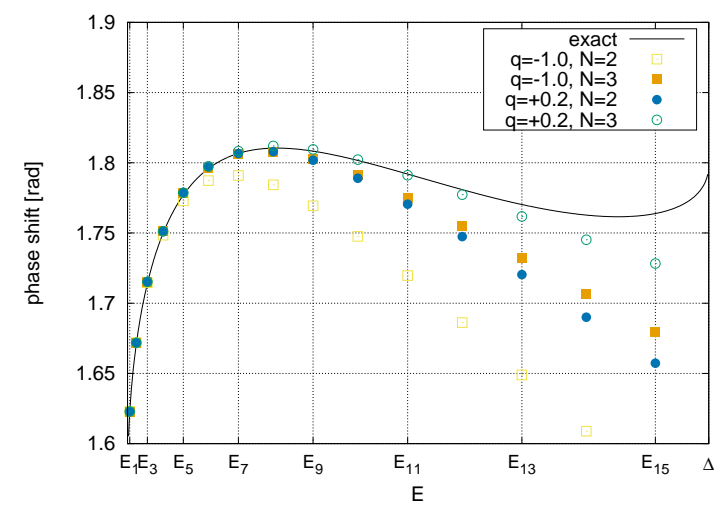

Figure 3: Phase shifts with $q=-1.0,+0.2$ and fixed $\rho=0.5$, at $N=2,3$.

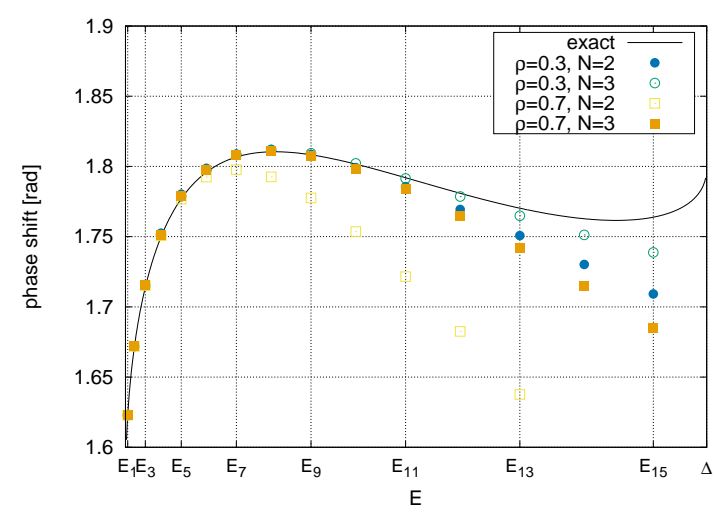

Figure 4: Phase shifts with fixed $q=+0.2$ and $\rho=$ $0.3,0.7$, at $N=2,3$.

\section{Summary}

We have studied the properties of the non-local potentials introduced by HAL QCD collaboration. We have employed an analytically solvable coupled-channel model to precisely perform the derivative expansion to higher orders, since it would require huge computational cost in lattice QCD. The generalized derivative expansion has been introduced to be applied to the present model, which involves unsmooth NBS wave functions. We have investigated the convergence of the generalized expansion by evaluating the validity of the truncation at a finite order.

We have observed that the generalized derivative expansion converges such that the agreement between the phase shift obtained from the HAL QCD potentials and the exact one becomes better for higher truncation orders. The result indicates that extrapolation to higher energies than those used as input is possible. We have also found that the convergence can be improved by either varying the choice of hadron interpolating fields or properly choosing the expansion scale which appears in the generalized derivative expansion.

\section{Acknowledgements}

We would like to thank K. Hiranuma, A. Hosaka, and the members of HAL QCD collaboration for helpful and enlightening discussions about this study. This work was supported by JSPS KAKENHI Grand Numbers JP25400244 and JP25247036, and by MEXT as "Priority Issue on Post-K computer" (Elucidation of the Fundamental Laws and Evolution of the Universe) and JICFuS.

\section{References}

[1] N. Ishii, S. Aoki and T. Hatsuda, Phys. Rev. Lett. 99, 022001 (2007).

[2] S. Aoki, T. Hatsuda, and N. Ishii, Prog. Theor. Phys. 123, 89 (2010).

[3] K. Murano, N. Ishii, S. Aoki, and T. Hatsuda, Prog. Theor. Phys. 125, 1225 (2011).

[4] M. C. Birse, [arXiv:1208.4807[nucl-th]].

[5] T. Sugiura et al., in preparation. 